\title{
Concurrent operation of 10 gyrotrons at W7-X - experience and improvement opportunities
}

\author{
H. Braune, K.J. Brunner, H. P. Laqua, S. Marsen, D. Moseev, F. Noke, F. Purps, N. Schneider,
} T. Schulz, T. Stange, P. Uhren, F. Wilde and the W7-X team at IPP, IGVP Stuttgart and KIT Karlsruhe Max-Planck-Institut für Plasmaphysik, Garching and Greifswald, Germany

\begin{abstract}
At the stellarator Wendelstein 7-X (W7-X) Electron Cyclotron Resonance Heating (ECRH) is the main heating system for steady-state operation and was the only available heating system during the operation phase OP1.2a in 2017. The ECRH, equipped with 10 operational $140 \mathrm{GHz}$ gyrotrons [1], was used for different tasks at W7-X such as wall conditioning, controlled plasma start-up from the neutral gas up to steady state plasma control and different heating scenarios such as X2-mode and O2-mode heating as well as current drive (ECCD). The operational experiences and improvement opportunities of the ECRH plant will be discussed.
\end{abstract}

\section{INTRODUCTION AND BACKGROUND}

$\mathrm{T}_{1}$ HE recent W7-X operation phase OP1.2a was performed with $10140 \mathrm{GHz}$ Gyrotrons in total. W7-X is currently equipped with an inertially cooled divertor with an input energy limit up to $80 \mathrm{MJ}$. The main goal of this operation phase was to explore and prepare operation scenarios for steady-state high performance operation in future campaigns, when $\mathrm{W} 7-\mathrm{X}$ is fully equipped with watercooled plasma facing components. The W7-X operation was powered by ECRH exclusively. The available total port through microwave power was more than 7.2 MW as shown in Fig. 1. Stationary discharges up to $30 \mathrm{~s}$ were achieved only limited by the maximum divertor energy load. The long discharges were also used to demonstrate current control and bootstrap current compensation by ECCD.

The ECRH transmission line system was supplemented by two remote steering launchers, an important concept for the future fusion reactor [2] which was tested at W7-X for the first time for plasma heating and current drive.

\section{PREPARATION OF THE ECRH INSTALLATION}

The individual power characteristics (acceleration voltage vs. output power) of each gyrotron were measured. Based on these power characteristics for the expected pulse length up to $30 \mathrm{~s}$, the W7-X main control real time system has only one parameter to control the output power of each gyrotron. By these means, the power variation capability during discharges is achieved.

The previous main power supply set up has the capability to provide 10 gyrotrons, two of them in parallel arrangement. The couple of gyrotron that operated in parallel was changed with the objective of equivalent output power at the same cathode voltage feed. Two gyrotrons with comparable behaviour pattern delivered by Thales Electron Devices (TED) were selected. The cathode voltage feed of different type gyrotrons separately, one delivered by TED and the other one delivered by Communications \& Power Industries (CPI), leads to a gain of $100 \mathrm{~kW}$ microwave output power at least per gyrotron compared to the first approach when these gyrotrons were operated in parallel based on their point of installation within the ECRH set up.
The modification was performed by changing of high voltage connection cables against each other due to the move of gyrotrons would require much more effort.

Three of the existing main power modules were equipped with a new controller in connection with new software release by the manufacturer Amperon. The voltage and current thresholds $(50 \mathrm{~A} / 65 \mathrm{kV})$ are replaced by a slightly higher power threshold (currents up to $70 \mathrm{~A}$ with $50 \mathrm{kV}$ output voltage will be achievable $->$ sufficient for $1.5 \mathrm{MW}$ gyrotron).

\section{HEATING SCENARIOS - ACHIEVED MAIN PLASMA PARAMETERS}

The W7-X plasmas were generated and sustained with the 2nd harmonic EC heating in the well-absorbed extraordinary polarization (X2) at densities below the X-mode cut off of $1.2 \times 10^{20} \mathrm{~m}^{-3}$. Current control and bootstrap current compensation by ECCD was demonstrated within the long discharges up to $30 \mathrm{~s}$. The rotational transform iota and thus the confinement and stability were intensely changed by localised ECCD.

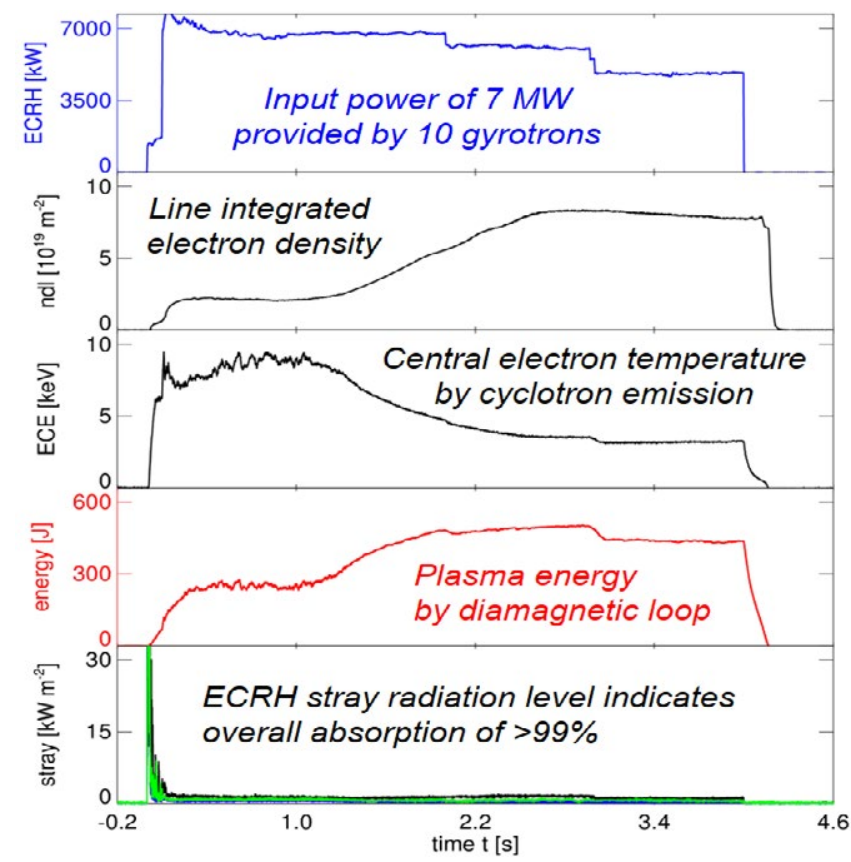

Fig. 1: Helium plasma discharge with 10 used gyrotrons, at around $2 s$ and $3 s$ the power stepped down by switching off of gyrotrons

In order to avoid beam reflection at densities close to the cut off and above that are relevant for a future fusion reactor, a dedicated triple beam path heating scenario with O-mode polarisation has been developed. Target plasma was generated by at least three gyrotrons with the X-mode polarisation. Afterwards, the remaining gyrotrons were switched on in O-mode and the start-up gyrotrons were changed to O-mode as well. A density of $1.4 \times 10^{20} \mathrm{~m}^{-3}$ was 
achieved in combination with pellet injection.

Due to the first path $\mathrm{O} 2$ mode absorption of only $70 \%$ a triple beam path was developed as shown in Fig. 2 based on individual holographic reflection tiles for each beam and a polished steel panel at the low field side. The $\mathrm{O} 2$ mode absorption was increased up to $90 \%$ with these three passes through the EC-resonance in the plasma centre.

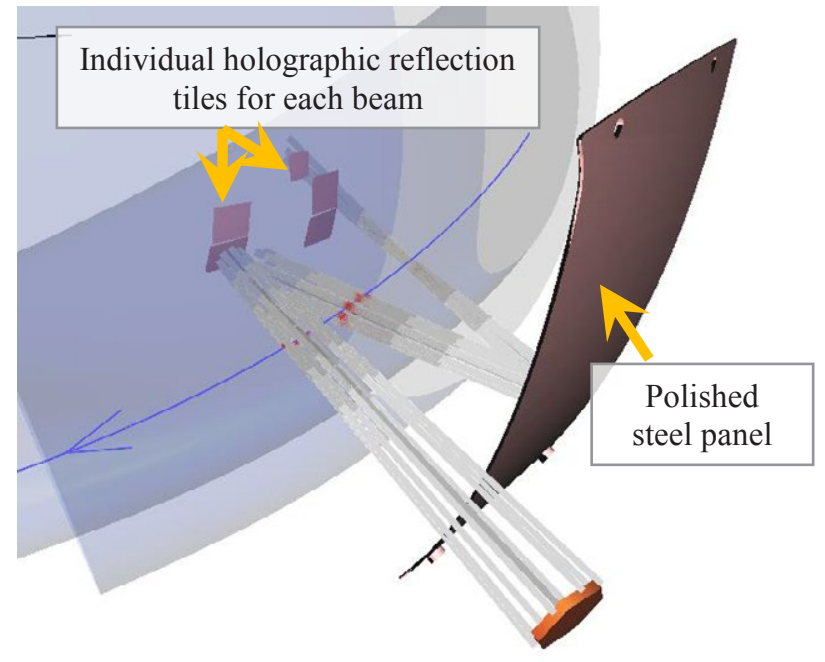

Fig. 2: Example of triple beam path for one (Alpha1) beamline

\section{NEW GYROTRON OPERATION MODES}

The W7-X gyrotrons were operated not at the maximum output power level during the previous operation phases in order to avoid gyrotron interlocks caused by instabilities.

A new software solution based on the fast gyrotron control system tries to keep the gyrotron in operation by acceleration voltage reduction for $500 \mu \mathrm{s}$. This solution [3] uses the capabilities of the fast control FPGA in connection with the achievable slew rates in the range of $0.4 \mathrm{kV} / \mu \mathrm{s}$ of the main and the body power supplies. The fast gyrotron control system monitors the microwave output power based on microwave sensitive diodes and if the output power drops below a certain threshold the gyrotron acceleration voltage would be reduced about $12 \mathrm{kV}$ as shown in Fig 3 for both high voltages separately.

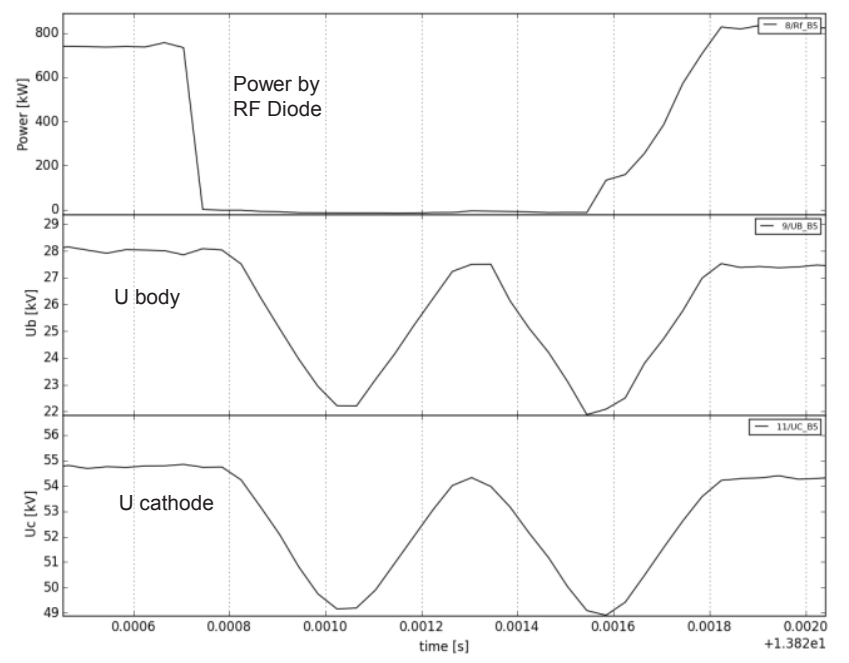

Fig. 3: RF power, body voltage, cathode voltage vs. time

Two fast acceleration voltage reduction cycles were required in this case to achieve approximately the same microwave output power as before by recovering the gyrotron working mode.

The gyrotron interlock system turns the gyrotron off if the output power does not come back within $2 \mathrm{~ms}$.

This kind of mode recovery allows a more reliable gyrotron operation close to the maximum output power limit. The gain of average power would be likely $100 \mathrm{~kW}$ per gyrotron.

\section{OUTLOOK}

The collector sweep system of the W7-X gyrotrons delivered by TED needs to improve in order to reduce the collector surface temperature slightly more as already achieved especially at the bottom region. This sweep system consists of two different coil arrangements, Vertical Field Sweep and Transverse Field Sweep, which are fed by single power supplies separately. A synchronised operation of both sweep power supplies will lead to a more smooth power distribution on the bottom of the collector surface and will avoid the shift of the electron strike line at the top of the collector toward to the cover plate that are not sufficiently cooled.

The W7-X ECRH installation offers the opportunity to enhance the amount of gyrotrons up to 12 . The main power supply upgrading is currently under construction. First of all the power supply is going to provide 12 gyrotrons simultaneously. Furthermore, a slightly higher power threshold replaces the voltage and current thresholds of $50 \mathrm{~A}$ and $65 \mathrm{kV}$. Currents up to $70 \mathrm{~A}$ with $50 \mathrm{kV}$ output voltage will be achievable and that are sufficient for prospective $1.5 \mathrm{MW}$ gyrotrons which are currently under development at different manufacturers [4] [5].

\section{REFERENCES}

[1] V. Erckmann et al. "ECRH and W7-X - an intriguing pair" Radio Frequency Power in Plasmas, Proc. 20 Topical Conference, Sorrento, Italy, AIP Conference Proceedings 1580 (2013) page 542

[2] W. Kasparek et al. "Remote-Steering Launchers for the ECRH system on the Stellarator W7-X", EPJ Web of Conferences 87, 04005 (2015)

[3] F. Wilde et al. "Measurements of Satellite Mode Activity and Automated Mode Recovery in $140 \mathrm{GHz}$ Wendelstein 7-X Gyrotrons", $20^{\text {th }}$ Joint Workshop on Electron Cyclotron Emission (ECE) and Electron Cyclotron Resonance Heating (ECRH), May 14-17, 2018 Greifswald, Germany, Book of abstracts page 24

[4] G. Denisov " New Developments of Megawatt Power Gyrotrons in Russia", $20^{\text {th }}$ Joint Workshop on Electron Cyclotron Emission (ECE) and Electron Cyclotron Resonance Heating (ECRH), May 14-17, 2018 Greifswald, Germany, Book of abstracts page 18

[5] J. Jelonek et al. "Towards Advanced Fusion Gyrotrons: 2018 Update on Activities within EUROfusion", $20^{\text {th }}$ Joint Workshop on Electron Cyclotron Emission (ECE) and Electron Cyclotron Resonance Heating (ECRH), May 14-17, 2018 Greifswald, Germany, Book of abstracts page 19

Acknowledgement:

This work has been carried out within the framework of the EUROfusion Consortium and has received funding from the Euratom research and training programme 2014-2018 under grant agreement No 633053. The views and opinions expressed herein do not necessarily reflect those of the European Commission. 